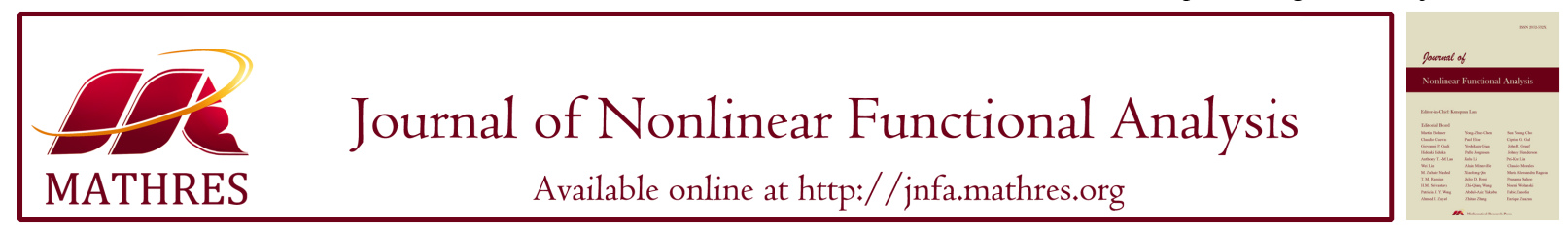

\title{
FIXED POINTS FOR RIGHT REVERSIBLE SEMIGROUPS SATISFYING THE CONTROLLABLE PUNCTUAL INEQUALITY
}

\author{
ABDELKADER DEHICI \\ Laboratory of Informatics and Mathematics, University of Souk-Ahras, Souk-Ahras 41000, Algeria
}

\begin{abstract}
In this paper, we give some fixed point results for right reversible semitopological semigroups, which satisfy the "controllable punctual inequality", on convex subsets of Banach spaces.
\end{abstract}

Keywords. Banach space; Semitopological semigroup; Right reversible semigroup; Invariant mean, Common fixed point.

\section{INTRODUCTION}

Throughout this paper, we assume that $X$ is a Banach space and $A$ is a nonempty subset of $X$ and use $\bar{A}$ and $\operatorname{co}(A)$ denote the closure and the convex hull of $A$ in $X$, respectively.

Let $S$ be a semitopological semigroup. That is, $S$ is a semigroup with a Hausdorff topology such that the mappings $s \in S \longrightarrow s t$ and $s \in S \longrightarrow t s$ are continuous from $S$ into $S$ for each $t \in S$. $S$ is called right reversible if any two closed left ideals of $S$ has non-void intersection. In this case, $(S, \leq)$ is a directed system when the binary relation " $\leq$ " on $S$ is given by $t_{1} \leq t_{2}$ if and only if $\left\{t_{1}\right\} \bigcup \overline{S t_{1}} \supseteq\left\{t_{2}\right\} \bigcup \overline{S t_{2}}, t_{1}, t_{2} \in S$ and $t_{1}<t_{2}$ means that $t_{1} \leq t_{2}$ and $t_{1} \neq t_{2}$. The family of right reversible semitopological semigroups contains in particular those of commutative semigroups and right amenable semitopological semigroups as discrete semigroups; see [1, 2, 3, 4, 5, 6, 7, 8, 9] and the references therein. By the same approach, we can define left reversible semigroups. $S$ is said to be reversible if $S$ is both left and right reversible. Let $l^{\infty}(S)$ be the Banach space of bounded real-valued functions on $S$ with the supremum norm. For $s \in S$ and $g \in l^{\infty}(S)$, the left and right translations of $g$ in $l^{\infty}(S)$ are defined by

$$
l_{s} g(t)=g(s t) \text { and } r_{s} g(t)=g(t s), \forall t \in S .
$$

Let $Z$ be a closed linear subspace of $l^{\infty}(S)$ containing constants and invariant under translations, i.e., $l_{s}(Z) \subset Z$ and $r_{s}(Z) \subset Z$. A linear functional $\mu \in Z^{\star}$ is called a left invariant mean on $Z$ if $\|\mu\|=\mu(1)=1$ and $\mu\left(l_{s} g\right)=\mu(g)$ for each $s \in S$ and $g \in Z$. In a similar way, we can define a right invariant mean. Let $C(S)$ be the closed subalgebra consisting of all continuous

E-mail address: dehicikader@yahoo.fr.

Received April 2, 2019; Accepted January 4, 2020.

(C)2020 Journal of Nonlinear Functional Analysis 
functions on $S$ and let $L U C(S)$ be the space of left uniformly continuous functions on $S$, i.e. all functions $f \in C(S)$ such that the mappings $s \in S \longrightarrow l_{s} f$ from $S$ to $C(S)$ are continuous if $C(S)$ is equipped with the sup norm topology. The space of right uniformly continuous functions on $S$ denoted by $R U C(S)$ can be defined by the same way. For more details, we refer to $[1,2,3,4,5,6,7,8,9,10]$, from which, we can find a number of results on fixed point theory of semigroup actions on convex subsets of Banach spaces and some convergence results. These results involve many problems in this direction which are related to the Bruck's conjecture [11], which asserts that the weak fixed point property (w-FPP) is equivalent to the weak fixed point property for left reversible semigroups.

Let $K$ be a nonempty subset of a Banach space $X$ with a norm $\|$.$\| . An action of S$ on $K$ is a mapping of the set $S \times K$ into $K$, denoted by $(s, x) \longrightarrow s \cdot x=T_{s} x$ for which $\left(s_{1} s_{2}\right) \cdot x=s_{1} \cdot\left(s_{2} \cdot x\right)$ for all $s_{1}, s_{2} \in S, x \in K$. The set of mappings $\mathscr{S}=\left(T_{s}, s \in S\right)$ is called a representation of $S$. Let $G \subseteq S$. A point $x \in K$ is a common fixed point of $G$ with respect to this action if $s . x=T_{s} x=x$ for all $s \in G$. The set of common fixed points of $G$ is denoted by $F(G)$. An action of $S$ on $K$ is called generalized nonexpansive if it satisfies that, for all $s \in S$ :

$$
\begin{aligned}
\|s . x-s . y\| & =\left\|T_{s} x-T_{s} y\right\| \\
& \leq \alpha_{1}\|x-y\|+\alpha_{2}\|x-s . x\|+\alpha_{3}\|y-s . x\|+\alpha_{4}\|y-s . y\|+\alpha_{5}\|x-s . y\|
\end{aligned}
$$

for all $x, y \in K$, where $\alpha_{i} \geq 0$ for all $i=1, \ldots, 5$ and $\sum_{i=1}^{5} \alpha_{i}=1$. If $\alpha_{i}=0$ for all $i=2, \ldots, 5$ (consequently $\alpha_{1}=1$ ), then the action is said to be nonexpansive. If, for each $x \in K$ and $s \in S$, the maps $s \longrightarrow T_{s} x$ from $S$ into $K$ and $x \longrightarrow T_{s} x$ from $K$ into $K$ are continuous, then the action is said to be separately continuous. In [1], Lau and Wong gave fixed point results for a generalized nonexpansive action on convex compact subsets of strictly convex Banach spaces and they asserted that these results hold for the case of Hausdorff locally convex topological spaces for which the topology is induced by a family of seminorms. Recently, Dehici [12] established some fixed point results for Kannan's actions $\left(\alpha_{1}=\alpha_{3}=\alpha_{5}=0, \alpha_{2}=\alpha_{4}=\frac{1}{2}\right)$ of weakly continuous mappings on weakly compact convex subsets of strictly convex Banach spaces.

\section{MAIN RESUlTS}

First, we give the following basic definition.

Definition 2.1. Let $S$ be a right reversible semitopological semigroup. Suppose that $S$ acts on a convex subset $C$ of a Banach space $X$. We say that $S$ satisfies "controllable punctual inequality" if $S$ has a minimal element $\min (S)$ satisfying that $T_{\min (S)}=I_{C}$ and for all $x \in C$ and all $\alpha, \beta \in S \backslash\{\min (S)\}$, there exists $\gamma(x) \in S \backslash\{\min (S)\}$ with $\gamma(x) \leq \alpha$ and $\gamma(x) \leq \beta$ such that

$$
\left\|T_{\alpha} x-T_{\beta} x\right\| \leq\left\|x-T_{\gamma(x)} x\right\|
$$

Let $n \geq 2$ be an integer. Under the notations of Definition 2.1, we denote

$$
\begin{aligned}
\Xi_{n}= & \{I \subset S, I \text { totally ordered, } \operatorname{card}(I)=n \text { and } \\
& \gamma(x) \in I \backslash\{\min (I)\} \text { for all } x \in C, \alpha, \beta \in I \backslash\{\min (I)\}\}
\end{aligned}
$$

For $I \in \Xi_{n}$, we define

$$
\mathfrak{I}_{n, I}=\left\{\widetilde{T}=\sum_{\alpha \in I} \beta_{\alpha} T_{\alpha}, \sum_{\alpha \in I} \beta_{\alpha}=1, T_{\min _{I}}=I_{C} \text { and } \beta_{\min (I \backslash\{\min I\})}>0\right\} .
$$


Our first result is given by the following theorem

Theorem 2.2. Let $S$ be a right reversible semitopological semigroup which acts on a convex subset $C$ of a Banach space X. If S satisfies a "controllable punctual inequality", then, for every integer $n>2$ and for all $I \in \Xi_{n}$, we have

$$
F\left(\mathfrak{I}_{n, I}\right)=F(I)
$$

Proof. It is easy to observe that $F(I) \subseteq F\left(\mathfrak{I}_{n, I}\right)$. Now, to prove the converse, we let $x_{0} \in F\left(\mathfrak{I}_{n, I}\right)$. For every $\widetilde{T} \in \mathfrak{I}_{n, I}$, we have $\widetilde{T}\left(x_{0}\right)=x_{0}$. Hence, there exists a finite set of positive real numbers $\left(\beta_{\alpha}\right) \subseteq[0,1]$ with $\sum_{\alpha \in I} \beta_{\alpha}=1$ and $\beta_{\min (I \backslash\{\min I\})}>0$ such that $T_{\min I}=I_{C}$ and $\widetilde{T}=\sum_{\alpha \in I} \beta_{\alpha} T_{\alpha}$. Thus

$$
\widetilde{T} x_{0}=\left(\sum_{\alpha \in I} \beta_{\alpha} T_{\alpha}\right) x_{0}=x_{0}
$$

which gives that

$$
x_{0}=\left(\sum_{\alpha \in I \backslash\{\min (I)\}}\left(\frac{\beta_{\alpha}}{1-\beta_{\min (I)}}\right) T_{\alpha}\right) x_{0} \quad\left(\beta_{\min (I)} \neq 1 \text { since } \beta_{\min (I \backslash\{\min (I)\})}>0\right) .
$$

Let

$$
\delta=\sup \left\{\left\|T_{\alpha} x_{0}-T_{\beta} x_{0}\right\|, \alpha, \beta \in I\right\} .
$$

Letting $\delta>0$, the fact that the action $S$ satisfies the "controllable punctual inequality" proves that there exists a minimal element $\gamma\left(x_{0}\right) \in I \backslash\{\min (I)\}$ such that

$$
\delta=\left\|x_{0}-T_{\gamma\left(x_{0}\right)} x_{0}\right\|
$$

Since

$$
\sum_{\alpha \in I \backslash\{\min (I)\}} \frac{\beta_{\alpha}}{1-\beta_{\min (I)}}=1
$$

one has

$$
\left.\left.x_{0}=\delta_{\mu} T_{\mu} x_{0}+\left(1-\delta_{\mu}\right) z, \quad \delta_{\mu} \in\right] 0,1\right]
$$

where

$$
z \in \operatorname{co}\left\{T_{\alpha} x_{0}, \alpha \in I \backslash\{\min (I), \min (I \backslash \min \{I\})\}\right\}
$$

and

$$
\mu=\min (I \backslash\{\min (I)\}) .
$$

Thus

$$
\begin{aligned}
\delta & =\left\|x_{0}-T_{\gamma\left(x_{0}\right)} x_{0}\right\| \\
& =\left\|\delta_{\mu} T_{\mu} x_{0}+\left(1-\delta_{\mu}\right) z-T_{\gamma\left(x_{0}\right)} x_{0}\right\| \\
& \leq \delta_{\mu}\left\|T_{\mu} x_{0}-T_{\gamma\left(x_{0}\right)} x_{0}\right\|+\left(1-\delta_{\mu}\right)\left\|z-T_{\gamma\left(x_{0}\right)} x_{0}\right\| \\
& \leq \delta_{\mu} \delta+\left(1-\delta_{\mu}\right) \delta \\
& =\delta .
\end{aligned}
$$

$(\iota):$ If $\gamma\left(x_{0}\right)=\mu$, this is a contradiction, since we obtain that $\left\|T_{\gamma\left(x_{0}\right)} x_{0}-T_{\gamma\left(x_{0}\right)} x_{0}\right\|=0=\delta$. 
$(\iota l)$ : If $\gamma\left(x_{0}\right)>\mu$, by the assumption $(*)$ and the definition of the family $\Xi_{n}$, we obtain the existence of $\theta \in I \backslash\{\min (I)\}$ with $\theta \leq \mu<\gamma\left(x_{0}\right)$ such that

$$
\delta \leq\left\|T_{\mu} x_{0}-T_{\gamma\left(x_{0}\right)} x_{0}\right\| \leq\left\|x_{0}-T_{\theta} x_{0}\right\|,
$$

which gives that $\left\|x_{0}-T_{\theta} x_{0}\right\|=\delta$ and contradicts the fact that $\gamma\left(x_{0}\right)$ is the minimal element $\alpha \in I \backslash\{\min (I)\}$ such that $\delta=\left\|x_{0}-T_{\alpha} x_{0}\right\|$. Necessarily, we get $\delta=0$ and $\left\|x_{0}-T_{\alpha} x_{0}\right\|=0$ for all $\alpha \in I$. Consequently $x_{0} \in F(I)$, which completes the proof.

Corollary 2.3. (see [13, Theorem 1]) Let $C$ be a convex subset of a Banach space $X$ and let $T$ be a nonexpansive self-mapping on $C$. Let $n \geq 1$ be an integer. Denote by $S_{n}=\sum_{i=0}^{n} \lambda_{i} T^{i}$ (with the notation $\left.T^{0}=I_{C}\right)$, where $\left(\lambda_{i}\right)_{i=0}^{n} \subset[0,1]$ with $\lambda_{1}>0$ and $\sum_{i=0}^{n} \lambda_{i}=1$. Then $F\left(S_{n}\right)=F(T)$.

Proof. The result follows from Theorem 2.2 by taking $S=\{0\} \cup \mathbb{N}$ and $I=\{0,1, \ldots ., n\}$. In this case, the action is given by $r . x=T^{r} x$ for all $x \in C$. The fact that this action satisfies the "controllable punctual inequality" is easy to check. Indeed, it suffices to observe that, for all integers $r, p \geq 1(p<r)$ and all $x \in C$, we have

$$
\left\|T^{r} x-T^{p} x\right\| \leq\left\|x-T^{r-p} x\right\| .
$$

Consequently, we can take $\gamma(x)=r-p$ for each $x \in C$.

Remark 2.4. In the definition of the family $\mathfrak{I}_{n, I}$, the assumption that $\beta_{\min (I \backslash\{\min I\})}>0$ is crucial to obtain the result of Theorem 2.2. In Corollary 2.3, this assumption is reduced to the fact that $\lambda_{1}>0$. To see this, let $T:[0,1] \longrightarrow[0,1]$ be given by $T(x)=1-x$. We have $F(T)=\left\{\frac{1}{2}\right\}$. Moreover, if we take the real polynomial $P(x)=\frac{1}{2}+\frac{1}{2} x^{2}$, then $F(P(T))=F\left(I_{[0,1]}\right)=[0,1]$, which implies that $F(T) \varsubsetneqq F(P(T))$, however it can be obtained that $F(P(T))=F\left(T^{2}\right)$.

Definition 2.5. Let $X$ be a Banach space and let $C, D$ be two nonempty subsets of $X$ such that $C \subset D$. A continuous mapping $P: D \longrightarrow C$ is called a retraction of $D$ onto $C$ if $P x=x$ for all $x \in C$.

The property of the sunny retraction and its characterization in smooth Banach spaces plays a crucial role in the theory of nonexpansive mappings. For investigation on fixed points involving sunny retractions, one refers to [14] and the references terein. In 1975, Baillon [15] first proved the following ergodic theorem which asserts that if $C$ is a closed convex subset of a Hilbert space and $T$ is a nonexpansive self-mapping on $C$ with $F(T) \neq \emptyset$, then, for each $x \in C$, the Cesaro mean

$$
S_{n}(x)=\frac{1}{n} \sum_{k=0}^{n-1} T^{k} x
$$

converges weakly to some $y \in F(T)$. As it was indicated in [8], if $P x=y$ for all $x \in C$, then $P$ is a nonexpansive retraction from $C$ to $F(T)$ such that $P T=T P=P$, and $P x \in \overline{c o}\left\{T^{n} x, n=\right.$ $1,2, \ldots \ldots\}$ for each $x \in C$. By Corollary 2.3, it is easy to conclude that $F\left(S_{n}\right)=F(T)$ for all integer $n>1$. In [16], Hirano and Takahashi obtained the existence of such retraction in Banach spaces and improved many results in the literature.

In the following result, we establish the existence of average mappings and retractions for the family of mappings $\mathfrak{I}_{n, I}$. 
Theorem 2.6. Let $X$ be a reflexive Banach space and let $C$ be a closed convex subset of $X$. Assume that $S$ is a right reversible semitopological semigroup of nonexpansive mappings which acts on $C$ for which $R U C(S)$ has a right invariant mean. If there exists an element in $C$ with a bounded orbit, then, for all integer $n \geq 2, \widetilde{T}_{t_{1}, t_{2}, \ldots, t_{n}}$ is a nonexpansive average mapping of $C$ such that $\widetilde{T}_{t_{1}, t_{2}, \ldots ., t_{n}} x \in \operatorname{co}\left\{T_{\alpha} x\right\}_{\alpha \in I, I \in \Xi_{n}}$ for all $x \in C$ and there exists a nonexpansive mapping $P$ on $C$ such that $\widetilde{T}_{t_{1}, t_{2}, \ldots, t_{n}} P=P$. Moreover, if $C$ has the normal structure and $S$ is reversible together with $P$ (given in [4, Theorem 6]) is affine, then $\widetilde{T}_{t_{1}, t_{2}, \ldots, t_{n}} P=P \widetilde{T}_{t_{1}, t_{2}, \ldots, t_{n}}=P$.

Proof. Denote

$$
\widetilde{T}_{t_{1}, t_{2}, \ldots ., t_{n}}=\sum_{t_{1}, \ldots \ldots, t_{n}} \beta_{t_{i}} T_{t_{i}}
$$

where $\sum_{t_{1}, \ldots \ldots, t_{n}} \beta_{t_{i}}=1$. It is easy to observe that $\widetilde{T}_{t_{1}, t_{2}, \ldots, t_{n}}$ is an average nonexpansive mapping. The existence of a retraction $P$ on $C$ such that $T_{S} P=P$ (see [4, Lemma 6]) for each $s \in S$ implies that $\widetilde{T}_{t_{1}, t_{2}, \ldots, t_{n}} P=P$. Now, if $P=r Q$ (see [4, Theorem 6]) is affine, then we infer that

$$
P\left(\sum_{t_{1}, \ldots \ldots, t_{n}} \beta_{t_{i}} T_{t_{i}}\right)=\sum_{t_{1}, \ldots \ldots, t_{n}} \beta_{t_{i}} P T_{t_{i}}=\left(\sum_{t_{1}, \ldots \ldots, t_{n}} \beta_{t_{i}}\right) P=P .
$$

This conclude the desired result immediately.

Remark 2.7. Observe that if the set $I \in \Xi_{n}$ has a bounded orbit in $C$, then the family $\mathfrak{I}_{n, I}$ has also a bounded orbit and $F(I)=F\left(\mathfrak{I}_{n, I}\right)$ even in the empty case if the action satisfies the controllable punctual inequality.

\section{SOME REMARKS AND QUESTIONS}

In the case of nonexpansive mappings, it is easy to construct an example $T$ for which the set $\left\{T^{n}, n \geq 1\right\}$ is infinite. To see this, it suffices to take $X=\mathbb{R}$ equipped with its usual norm and $T: \mathbb{R} \longrightarrow \mathbb{R}$ given by $T x=\sqrt{x^{2}+1}$. A sample calculation shows that $T$ is nonexpansive. In additiona, we have $T^{n} x=\sqrt{x^{2}+n}$ for all $n \geq 2$. Thus, $T^{n} \neq T^{m}$ for all integers $n, m$ with $n \neq m$.

Let $X$ be a Banach space and let $C$ be a nonempty subset of $X$. Define the family $\Sigma_{C}$ of self-mappings on $C$ by

$$
\sum_{C}=\{T: C \longrightarrow C\}
$$

satisfying

$$
\|T x-T y\| \leq \frac{\|T x-x\|\|x-T y\|+\|T y-y\|\|y-T x\|}{\|T x-y\|+\|T y-x\|}, \quad x, y \in C, x \neq y
$$

with the additional assumption that $\|T x-y\|+\|T y-x\| \neq 0$. It is easy to observe that $\sum_{C}$ is a nonempty family since it contains constant mappings. It is shown that, for all $T \in \sum_{C}$ and for all $x \in C$, if $n, m \geq 1$ are arbitrary integers with $n \neq m$, we have $\left\|T^{n} x-T^{m} x\right\| \leq\|x-T x\|$ (see [17]).

Finally, we conclude our paper by the following interesting question and remark.

Question. Is it possible to find a Banach space $X$ and a nonempty subset $C$ of $X$ such that, for all $T \in \Sigma_{C}$ and for all integer $n \geq 2$, we have $T^{n} \in \Sigma_{C}$ and the set $\left\{T^{n}\right\}_{n \geq 1}$ of mappings is infinite.

Conclusion. The results presented in this paper were obtained for not necessarily commutative right reversible semigroups. We extended in particular those established in [18] for the case of 
a finite family of mappings and gave a perspective to investigate the problem of image recovery [19] for the right reversible semigroups.

\section{Acknowledgements}

The initial ideas of this paper were developed during the author's short academic stay at the Department of Mathematical and Statistical Sciences, University of Alberta, Canada (November 22-December 7, 2017). The author would like to thank Professor Anthony To-Ming Lau for his interest with great admiration and respect.

\section{REFERENCES}

[1] A. T. Lau, C. S. Wong, Common fixed points for semigroups of mappings, Proc. Amer. Math. Soc. 41 (1973), 223-228.

[2] A. T. Lau, W. Takahashi, Invariant means and fixed point properties for nonexpansive representations of topological semigroups, Topol. Methods. Nonlinear Anal. 5 (1995), 39-57.

[3] A. T. Lau, W. Takahashi, Invariant means and semigroups of nonexpansive mappings on uniformly convex Banach spaces, J. Math. Anal. Appl. 153 (1990), 497-505.

[4] A. T. Lau, W. Takahashi, Weak convergence and nonlinear ergodic theorems for reversible semigroups of nonexpansive mappings, Pacific J. Math. 126 (1987), 277-294.

[5] A. T. Lau, Y. Zhang, Fixed point properties for semigroups of nonlinear mappings and amenability, J. Funct. Anal. 263 (2012), 2949-2977.

[6] A. T. Lau, Invariant means on almost periodic functions and fixed point properties, Rocky Mountain J. Math. 3 (1973), 69-76.

[7] A. T. Lau, Normal structure and common fixed point properties for semigroups of nonexpansive mappings in Banach spaces, Fixed Point Theory Appl. 2010 (2010), Article ID 580956.

[8] A. T. Lau, Invariant means and fixed point properties of semigroup of nonexpansive mappings, Taiwanese J. Math. 12 (2008), 1525-1542.

[9] M. Neufang, J. Pachl, J. Steprāns, Group actions whose space of invariant means is finite dimensional, J. Nonlinear Var. Anal. 1 (2017), 307-319.

[10] W. Bartoszek, Nonexpansive actions of topological semigroups on strictly convex Banach spaces and fixed points, Proc. Amer. Math. Soc. 104 (1988), 809-811.

[11] R. E. Bruck, Jr, A common fixed point theorem for a commuting family of nonexpansive mappings, Pacific J. Math. 53 (1974), 59-71.

[12] A. Dehici, Common Fixed point theorems for semigroup actions of Kannan's type on strictly convex Banach spaces, J. Fixed. Point. Theory. Appl. 20 (2018), 100.

[13] W. A. Kirk, On successive approximations for nonexpansive mappings in Banach spaces, Glasgow Math. J. 12 (1971), 6-9.

[14] S. Reich, Asymptotic behavior of contractions in Banach spaces, J. Math. Anal. Appl. 44 (1973), 57-70.

[15] J. B. Baillon, Un théorème de type ergodique pour les contractions non linéaires dans un espace de Hilbert, C.R.Acad. Sci. Paris. Sér A-B. 280 (1975), 1511-1514.

[16] N. Hirano, W. Takahashi, Nonlinear ergodic theorems for an amenable semigroup of nonexpansive mappings in a Banach space, Pacific J. Math. 112 (1984), 333-346.

[17] B. K. Ray, S. P. Singh, Fixed point theorems in Banach spaces, Indian. J. Pure. Appl. Math. 9 (1978), 216-221.

[18] A. Dehici, Some remarks on generalized Kirk's process in Banach spaces and application, Turkish. J. Math. 43 (2019), 1395-1406.

[19] S. Kitahara, W. Takahashi, Image recovery by convex combinations of sunny nonexpansive retractions, Topol. Methods Nonlinear Anal. 2 (1993), 333-342. 